\title{
Very high energy $\gamma$-ray observations of the binary PSR B1259-63/SS2883 around the 2007 Periastron
}

F. Aharonian ${ }^{1,13}$, A. G. Akhperjanian ${ }^{2}$, G. Anton ${ }^{16}$, U. Barres de Almeida ${ }^{8, \star}$, A. R. Bazer-Bachi ${ }^{3}$, Y. Becherini ${ }^{12}$, B. Behera ${ }^{14}$, K. Bernlöhr ${ }^{1,5}$, A. Bochow ${ }^{1}$, C. Boisson ${ }^{6}$, J. Bolmont $^{19}$, V. Borrel ${ }^{3}$, J. Brucker ${ }^{16}$, F. Brun ${ }^{19}$, P. Brun ${ }^{7}$, R. Bühler ${ }^{1}$, T. Bulik ${ }^{24}$, I. Büsching ${ }^{9}$, T. Boutelier ${ }^{17}$, P. M. Chadwick ${ }^{8}$, A. Charbonnier ${ }^{19}$, R. C. G. Chaves ${ }^{1}$, A. Cheesebrough ${ }^{8}$, L.-M. Chounet ${ }^{10}$, A. C. Clapson ${ }^{1}$, G. Coignet $^{11}$, M. Dalton ${ }^{5}$, M. K. Daniel ${ }^{8}$, I. D. Davids ${ }^{22,9}$, B. Degrange ${ }^{10}$, C. Deil ${ }^{1}$, H. J. Dickinson ${ }^{8}$, A. Djannati-Atai ${ }^{12}$, W. Domainko ${ }^{1}$, L. O'C. Drury ${ }^{13}$, F. Dubois ${ }^{11}$, G. Dubus ${ }^{17}$, J. Dyks ${ }^{24}$, M. Dyrda ${ }^{28}$, K. Egberts ${ }^{1}$, D. Emmanoulopoulos ${ }^{14}$, P. Espigat ${ }^{12}$, C. Farnier ${ }^{15}$, F. Feinstein ${ }^{15}$, A. Fiasson ${ }^{11}$, A. Förster ${ }^{1}$, G. Fontaine ${ }^{10}$, M. Füßling ${ }^{5}$, S. Gabici ${ }^{13}$, Y. A. Gallant ${ }^{15}$, L. Gérard ${ }^{12}$, D. Gerbig ${ }^{21}$, B. Giebels ${ }^{10}$, J. F. Glicenstein ${ }^{7}$, B. Glück ${ }^{16}$, P. Goret ${ }^{7}$, D. Göring ${ }^{16}$, D. Hauser ${ }^{14}$, M. Hauser ${ }^{14}$, S. Heinz ${ }^{16}$, G. Heinzelmann ${ }^{4}$, G. Henri ${ }^{17}$, G. Hermann ${ }^{1}$, J. A. Hinton ${ }^{25}$, A. Hoffmann ${ }^{18}$, W. Hofmann ${ }^{1}$, M. Holleran ${ }^{9}$, S. Hoppe ${ }^{1}$, D. Horns ${ }^{4}$, A. Jacholkowska ${ }^{19}$, O. C. de Jager ${ }^{9}$, C. Jahn ${ }^{16}$, I. Jung ${ }^{16}$, K. Katarzyński ${ }^{27}$, U. Katz ${ }^{16}$, S. Kaufmann ${ }^{14}$, M. Kerschhaggl ${ }^{5}$, D. Khangulyan ${ }^{1}$, B. Khélifi ${ }^{10}$, D. Keogh ${ }^{8}$, D. Klochkov ${ }^{18}$, W. Kluźniak ${ }^{24}$, T. Kneiske ${ }^{4}$, Nu. Komin ${ }^{7}$, K. Kosack ${ }^{1}$, R. Kossakowski ${ }^{11}$, G. Lamanna ${ }^{11}$, J.-P. Lenain ${ }^{6}$, T. Lohse ${ }^{5}$, V. Marandon ${ }^{12}$, O. Martineau-Huynh ${ }^{19}$, A. Marcowith ${ }^{15}$, J. Masbou ${ }^{11}$, D. Maurin ${ }^{19}$, T. J. L. McComb ${ }^{8}$, M. C. Medina ${ }^{6}$, R. Moderski ${ }^{24}$, E. Moulin ${ }^{7}$, M. Naumann-Godo ${ }^{10}$, M. de Naurois ${ }^{19}$, D. Nedbal ${ }^{20}$, D. Nekrassov ${ }^{1}$, B. Nicholas ${ }^{26}$, J. Niemiec ${ }^{28}$, S. J. Nolan ${ }^{8}$, S. Ohm ${ }^{1}$, J.-F. Olive ${ }^{3}$, E. de Oña Wilhelmi ${ }^{1,12,29}$, K. J. Orford ${ }^{8}$, M. Ostrowski ${ }^{23}$, M. Panter ${ }^{1}$, M. Paz Arribas ${ }^{5}$, G. Pedaletti ${ }^{14}$, G. Pelletier ${ }^{17}$, P.-O. Petrucci ${ }^{17}$, S. Pita ${ }^{12}$, G. Pühlhofer ${ }^{18,14}$, M. Punch ${ }^{12}$, A. Quirrenbach ${ }^{14}$, B. C. Raubenheimer ${ }^{9}$, M. Raue ${ }^{1,29}$, S. M. Rayner ${ }^{8}$, M. Renaud ${ }^{12,1}$, F. Rieger ${ }^{1,29}$, J. Ripken ${ }^{4}$, L. Rob ${ }^{20}$, S. Rosier-Lees ${ }^{11}$, G. Rowell ${ }^{26}$, B. Rudak ${ }^{24}$, C. B. Rulten ${ }^{8}$, J. Ruppel ${ }^{21}$, V. Sahakian ${ }^{2}$, A. Santangelo ${ }^{18}$, R. Schlickeiser ${ }^{21}$, F. M. Schöck ${ }^{16}$, U. Schwanke ${ }^{5}$, S. Schwarzburg ${ }^{18}$, S. Schwemmer ${ }^{14}$, A. Shalchi ${ }^{21}$, M. Sikora ${ }^{24}$, J. L. Skilton ${ }^{25}$, H. Sol ${ }^{6}$, D. Spangler ${ }^{8}$, Ł. Stawarz ${ }^{23}$, R. Steenkamp ${ }^{22}$, C. Stegmann ${ }^{16}$, F. Stinzing ${ }^{16}$, G. Superina ${ }^{10}$, A. Szostek ${ }^{23,17}$, P. H. Tam ${ }^{14}$, J.-P. Tavernet ${ }^{19}$, R. Terrier ${ }^{12}$, O. Tibolla ${ }^{1}$, M. Tluczykont ${ }^{4}$, C. van Eldik ${ }^{1}$, G. Vasileiadis ${ }^{15}$, C. Venter ${ }^{9}$, L. Venter ${ }^{6}$, J. P. Vialle ${ }^{11}$, P. Vincent ${ }^{19}$, M. Vivier ${ }^{7}$, H. J. Völk ${ }^{1}$, F. Volpe ${ }^{1}$, S. J. Wagner ${ }^{14}$, M. Ward ${ }^{8}$, A. A. Zdziarski ${ }^{24}$, and A. Zech ${ }^{6}$

(Affiliations can be found after the references)

Received 16 April 2009 / Accepted 1 September 2009

\section{ABSTRACT}

\begin{abstract}
Aims. This article presents very-high-energy (VHE; $E>100 \mathrm{GeV}$ ) data from the $\gamma$-ray binary PSR B1259-63 as taken during the years 2005 , 2006 and before as well as shortly after the 2007 periastron passage. These data extend the knowledge of the lightcurve of this object to all phases of the 3.4 year binary orbit. The lightcurve constrains physical mechanisms present in this $\mathrm{TeV}$ source.

Methods. Observations of VHE $\gamma$-rays with the HESS telescope array using the Imaging Atmospheric Cherenkov Technique were performed. The HESS instrument features an angular resolution of $<0.1^{\circ}$ and an energy resolution of $<20 \%$. Gamma-ray events in an energy range of $0.5-70 \mathrm{TeV}$ were recorded. From these data, energy spectra and lightcurve with a monthly time sampling were extracted.

Results. VHE $\gamma$-ray emission from PSR B1259-63 was detected with an overall significance of 9.5 standard deviations using $55 \mathrm{~h}$ of exposure, obtained from April to August 2007. The monthly flux of $\gamma$-rays during the observation period was measured, yielding VHE lightcurve data for the early pre-periastron phase of the system for the first time. No spectral variability was found on timescales of months. The spectrum is described by a power law with a photon index of $\Gamma=2.8 \pm 0.2_{\text {stat }} \pm 0.2_{\text {sys }}$ and flux normalisation $\Phi_{0}=\left(1.1 \pm 0.1_{\text {stat }} \pm 0.2_{\text {sys }}\right) \times 10^{-12} \mathrm{TeV}^{-1} \mathrm{~cm}^{-2} \mathrm{~s}^{-1}$.

PSR B1259-63 was also monitored in 2005 and 2006, far from periastron passage, comprising $8.9 \mathrm{~h}$ and $7.5 \mathrm{~h}$ of exposure, respectively. No significant excess of $\gamma$-rays is seen in those observations.

Conclusions. PSR B1259-63 has been re-confirmed as a variable TeV $\gamma$-ray emitter. The firm detection of VHE photons emitted at a true anomaly $\theta \approx-0.35$ of the pulsar orbit, i.e. already $\sim 50$ days prior to the periastron passage, disfavors the stellar disc target scenario as a primary emission mechanism, based on current knowledge about the companion star's disc inclination, extension, and density profile.
\end{abstract}

Key words. radiation mechanisms: non-thermal - methods: observational - stars: binaries: general - stars: neutron - gamma rays: observations telescopes

* Supported by CAPES Foundation, Ministry of Education of Brazil. 
Table 1. System parameters of the binary system PSR B1259-63/SS2883 (Tavani \& Arons 1997).

\begin{tabular}{cc}
\hline \hline Parameter & Value \\
\hline Distance, $D[\mathrm{kpc}]$ & 1.5 \\
Eccentricity, $e$ & 0.87 \\
Orbital Period, $P_{\text {orb }}[\mathrm{d}]$ & 1273 \\
Last Periastron Passage & $2007-07-27$ \\
Compact Object & \\
Pulsar Period, $P[\mathrm{~ms}]$ & 47.7 \\
$\dot{P}=\frac{\mathrm{d} P}{\mathrm{~d} t}\left[\frac{\mathrm{S}}{\mathrm{s}}\right]$ & $2.27579 \times 10^{-15}$ \\
Spin-down age, $\tau[\mathrm{yr}]$ & $3 \times 10^{5}$ \\
Spin-down luminosity, $L_{p}\left[\mathrm{erg} \mathrm{s}{ }^{-1}\right]$ & $8 \times 10^{35}$ \\
Magnetic field, $B[\mathrm{G}]$ & $3 \times 10^{11}$ \\
Companion Star $(\mathbf{B} 2$ Ve) & \\
Temperature $[\mathrm{K}]$ & $10^{4}$ \\
Mass $\left[M_{\odot}\right]$ & 10 \\
\hline
\end{tabular}

\section{Introduction}

The binary system PSR B1259-63/SS2883 is known since its discovery at radio wavelengths by Johnston and collaborators in 1991 (Johnston et al. 1992) (see Table 1). It consists of a $48 \mathrm{~ms}$ pulsar with a Crab-like pulse profile (double peaked, showing a main pulse and an interpulse) in a 3.4 year eccentric orbit $(e=0.87)$ around a massive Be star. The latter feeds a dense circumstellar disc, as indicated by its optical spectrum and by the eclipse of the pulsed radio signal around periastron (Johnston et al. 1999). This circumstellar disc is likely to be misaligned with respect to the pulsar orbit (Melatos et al. 1995; Johnston et al. 2005; Bogomazov 2005). PSR B1259-63 has been observed in various energy bands from radio to $\gamma$-rays throughout the years since its discovery. Most observations took place around periastron passage where the distance between the pulsar and the massive star is at its minimum of $\sim 0.7 \mathrm{AU}$ and the interactions between the two objects are believed to be most intense.

X-ray observations of PSR B1259-63 show unpulsed emission with a variable flux and spectral index (Greiner et al. 1995; Hirayama et al. 1999; Nicastro et al. 1999; Shaw et al. 2004; Chernyakova et al. 2006). The X-ray luminosity in the $1-10 \mathrm{keV}$ band evolves significantly with orbital phase (mean anomaly), with $L_{\mathrm{X}} \sim 0.5 \times 10^{33} \mathrm{erg} \mathrm{s}^{-1}$ at apastron but reaching $L_{\mathrm{X}} \sim$ $10-20 \times 10^{33} \mathrm{erg} \mathrm{s}^{-1}$ some 10 days prior to and during periastron passage. The X-ray luminosity then decreases. The spectral index also shows orbit-related variability with softer indices at periastron just like the unpulsed radio flux which is enhanced during the passage (Neronov \& Chernyakova 2007). Recently, evidence for the presence of a spectral break around $\sim 5 \mathrm{keV}$ during the hard-spectrum state of the 2007 periastron passage was presented (Uchiyama et al. 2009).

PSR B1259-63 was first detected in $\mathrm{TeV} \gamma$-rays around its periastron passage in 2004 by HESS, making it the first known binary to emit at very high energies and the first variable VHE source in our Galaxy (Aharonian et al. 2005b). The HESS observations showed that the flux of VHE $\gamma$-rays from this source is significantly variable on timescales of days. The overall spectrum of the emission extracted from $49.8 \mathrm{~h}$ (livetime) of data followed a simple power law with photon index $\Gamma=2.7 \pm 0.2_{\text {stat }} \pm 0.2_{\text {sys }}$ and flux normalisation $\Phi_{0}=(1.3 \pm$ $\left.0.1_{\text {stat }} \pm 0.3_{\text {sys }}\right) \times 10^{-12} \mathrm{TeV}^{-1} \mathrm{~cm}^{-2} \mathrm{~s}^{-1}$ showing no indication for index variability on timescales of months. Unlike the three other binary systems LS5039 (Aharonian et al. 2005a), LSI+61 303
(Albert et al. 2006) and Cygnus X-1 (Albert et al. 2007) seen in the VHE domain, PSR B 1259-63 represents the only known system where the compact object is unambiguously identified as a neutron star (NS) (Johnston et al. 1992). The relatively short pulsar spin period and high spin-down luminosity (see Table 1) are sufficient to generate a relativistic pulsar wind (PW) which prevents accretion onto the neutron star. PSR B1259-63 can be classified as a binary system with a plerionic component (Tavani \& Arons 1997), i.e. as a unique system for the study of PW interactions with ambient radiation and matter outflow originating from a companion star.

The peculiar double humped shape of the VHE flux in 2004 as well as the correlated rise and fall in the lightcurves in other wavebands around periastron have been taken as hints for the causal influence of the Be star disc on the emission process (Kawachi et al. 2004; Chernyakova et al. 2006). The dense equatorial matter outflow is an ideal source of target material within the framework of a hadronic scenario where the ultrarelativistic PW particles would produce $\pi^{0}$ mesons and hence TeV $\gamma$-rays. However, the situation is ambiguous and such a scenario cannot be proven yet as the combined spectral and lightcurve data allow for different model explanations (Neronov \& Chernyakova 2007). The generation of TeV $\gamma$-rays within an Inverse Compton (IC) scenario is another explanation for the data (Kirk et al. 1999; Ball \& Kirk 2000; Dubus 2006a; Khangulyan et al. 2007; Sierpowska-Bartosik \& Bednarek 2008). In such models PW electrons moving with highly relativistic energies upscatter soft UV photons stemming from the stellar radiation field into the VHE regime. Strong constraints on the models can be obtained from phase dependent observations. Most relevant physical quantities such as the magnetic field, the radiation and matter densities and the binary separation are a function of the pulsar orbital phase. The changing geometry of the system as seen by the observer can also cause variations in the measured $\mathrm{TeV}$ flux (e.g. because of the anisotropic nature of IC scattering).

The previously published VHE lightcurve lacks data from the early phases prior to periastron. This prompted observation of PSR B1259-63 around the 2007 periastron (27th of July), the results of which are described here. A specific campaign was carried out from April to August 2007, resulting in $52.5 \mathrm{~h}$ (livetime) of data. Note that right at periastron passage PSR B1259-63 was not visible for HESS during night time. In addition, monitoring observations performed during the years 2005 ( $8.3 \mathrm{~h}$ livetime from March to April) and 2006 (6.9 h livetime from April to May) are also reported here.

\section{Observations and analysis}

The High Energy Stereoscopic System HESS is an array of four imaging air Cherenkov telescopes located in the Khomas Highland in Namibia $\left(23^{\circ} 16^{\prime} 17^{\prime \prime} \mathrm{S} 16^{\circ} 29^{\prime} 58^{\prime \prime} \mathrm{E}\right)$ at an altitude of $1800 \mathrm{~m}$ above sea level. Each telescope consists of a spherical dish $13 \mathrm{~m}$ in diameter, hosting 380 individual mirrors giving an overall reflective area of $107 \mathrm{~m}^{2}$. Cherenkov radiation as generated in extended air showers is collected by the mirrors and focused onto a camera consisting of 960 photomultipliers with a pixel size of $0.16^{\circ}$ resulting in a Field of View (FoV) of $\sim 5^{\circ}$. Following the usual trigger criterion with respect to telescope multiplicity for coincident operation, a shower image will be recorded once at least two out of four telescopes trigger (Funk et al. 2004). Determination of shower parameters and consequent evaluation of the primary particle type, energy and direction is done using an image reconstruction technique based on Hillas moments (Aharonian et al. 2006). The 
Table 2. Datasets for the HESS campaigns on PSR B1259-63/SS2883 in 2005, 2006 and 2007.

\begin{tabular}{lcccccccccc}
\hline Period & $\tau /[\mathrm{d}]$ & $\theta$ & $\phi$ & $t /[\mathrm{h}]$ & $N_{\mathrm{ON}}$ & $N_{\text {OFF }}$ & $\alpha$ & $N_{\gamma}$ & $S_{\text {Hillas }}^{*} /[\sigma]$ & $S_{\text {Model }}^{* *} /[\sigma]$ \\
\hline 2005 & 386 & 0.47 & 0.31 & 8.3 & 347 & 6909 & 0.047 & 22.7 & 1.2 & 1.2 \\
2006 & -454 & -0.48 & -0.37 & 6.9 & 271 & 3201 & 0.079 & 18.0 & 1.1 & 0.5 \\
\hline 2007 & & & & & & & & & & \\
April & -104 & -0.40 & -0.084 & 5.3 & 205 & 2571 & 0.074 & 15.8 & 1.1 & 2.9 \\
May & -74 & -0.38 & -0.060 & 14.6 & 623 & 7280 & 0.074 & 84.4 & 3.4 & 5.8 \\
June & -46 & -0.35 & -0.037 & 15.5 & 843 & 8373 & 0.073 & 228.8 & 8.4 & 9.3 \\
July & -17 & -0.25 & -0.014 & 14.4 & 575 & 6238 & 0.077 & 96.4 & 4.1 & 7.4 \\
August & 9 & 0.17 & 0.007 & 3.2 & 124 & 1383 & 0.073 & 22.9 & 2.1 & 0.9 \\
\hline 2007 Total & $\ldots$ & $\ldots$ & $\ldots$ & 52.5 & 2353 & 25633 & 0.074 & 448.7 & 9.5 & 13.2 \\
\hline
\end{tabular}

Shown are the days relative to periastron $\tau$, the true anomaly $\theta$, the orbital phase (mean anomaly) $\phi$, the livetime $t$, the number of ON and OFF events $N_{\mathrm{ON}}, N_{\mathrm{OFF}}$, the background normalisation $\alpha$ and the number of excess photons $N_{\gamma}$ for the Hillas type analysis for each observation period. The significances $S$ according to Li \& Ma (1983) are shown for both analysis techniques, as outlined in the text.

* Standard HESS analysis based on Hillas moments.

** HESS semi-analytical model analysis used as cross check.

HESS instrument has a trigger threshold for the photon energy of $\sim 100 \mathrm{GeV}$ for observations at zenith given an optical mirror efficiency of $>80 \%$. Above this ideal threshold a point source at zenith with a photon flux of $\sim 1 \%$ of that of the Crab nebula, i.e. $<2.0 \times 10^{-13} \mathrm{~cm}^{-2} \mathrm{~s}^{-1}$, can be detected in a $25 \mathrm{~h}$ observation at a significance level of $5 \sigma$.

Table 2 summarizes the dates and livetimes of the dataset used here. The data were taken in wobble-mode, i.e. with the pointing position slightly offset from the target position. Due to the radial acceptance profile of the detector this mode allows a simple simultaneous background estimation using the Reflected-Region method (Berge et al. 2007). For the observation campaign in 2007 this technique has been applied only with respect to right ascension (RA) at an offset of $0.7^{\circ}$ so as not to interfere with a second source in the FoV HESS J1303-631 (Aharonian et al. 2005c) which is located $\sim 0.6^{\circ}$ to the north of PSR B1259-63. The 2007 observations were carried out at zenith angles (ZA) ranging from $40.6^{\circ}$ to $62.7^{\circ}$. The mean ZA is $44^{\circ}$ leading to an analysis energy threshold of $620 \mathrm{GeV}$. The datasets from 2005 and 2006 contain exposures taken during the HESS galactic plane scan and dedicated observation runs on HESS J1303-631 with a positive offset of $0.7^{\circ}$ in declination (Dec.). This leads to wobble offsets with respect to the position of PSR B1259-63 for the analysis presented here both in RA and Dec., ranging between $0.5^{\circ}$ and $1.9^{\circ}$. The mean ZA for these observations were $44.1^{\circ}$ in 2005 and $47.1^{\circ}$ in 2006. The usual quality criteria with respect to weather conditions and fully functional array hardware are applied to the data (Aharonian et al. 2006).

\subsection{Detection}

The Hillas analysis was applied, incorporating so-called standard cuts on image quality (image amplitude 80 p.e.) and an angular cut of $\theta^{2}<0.0125$ for $\theta$ defined as angular distance between a $\gamma$-ray-like event and the nominal target position. In order to measure the hadronic background in the FoV simultaneously, the Reflected-Region background model was used. Using this technique, the level of OFF events is taken from regions within the same FoV which are located at the same distance with respect to the camera center as the ON region in order to account for the radial acceptance of the detector. OFF regions that overlapped with a circular region of radius $0.45^{\circ}$

\footnotetext{
${ }^{1}$ Higher wobble offsets $\left(>0.9^{\circ}\right)$ additionally increase the energy threshold due to the radial acceptance profile of the camera.
}

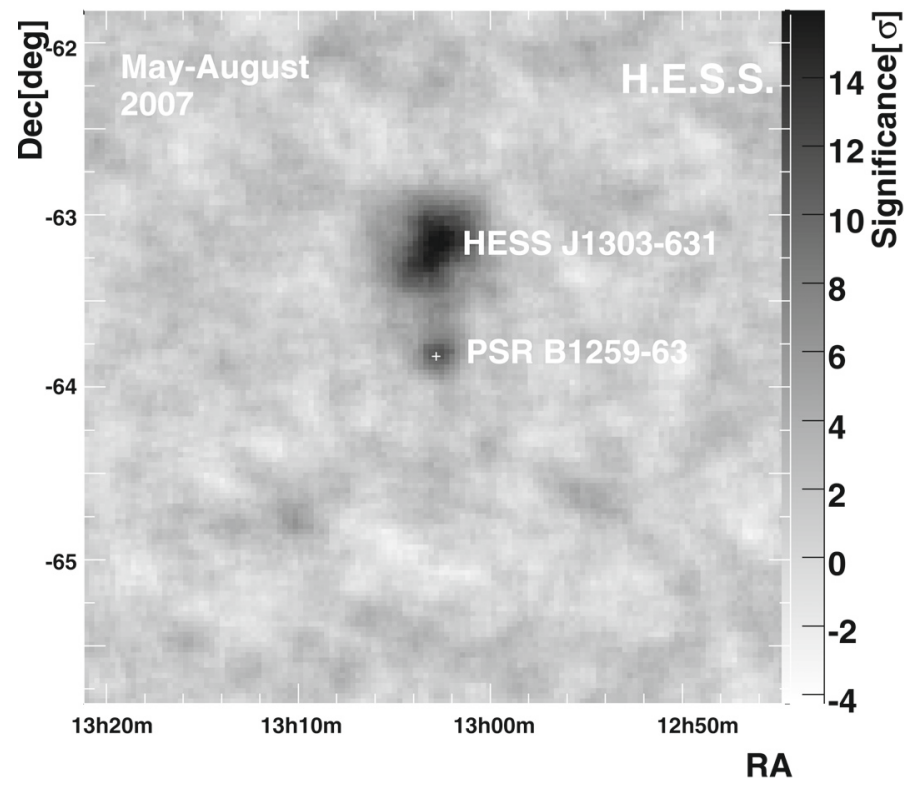

Fig. 1. Correlated significance map for the PSR B1259-63 FoV between May and August 2007. There is a clear signal in $\mathrm{TeV} \gamma$-rays visible from the target direction. The white cross indicates the nominal position of PSR B1259-63.The extended source to the north is HESS J1303-631 (Aharonian et al. 2005c).

at the position of HESS J1303-631 were omitted in the background calculation. A correction due to a reduced reflectivity in the instrument's mirrors has been taken into account as described in Aharonian et al. (2006). This standard HESS point source analysis for the 2007 dataset resulted in a clear excess of 450 photons coming from the direction of PSR B1259-63 resulting in a statistical significance following Li \& Ma (1983) of 9.5 standard deviations for this detection (see Table 2). Figure 1 shows the correlated significance map from May to August 2007 for the PSR B1259-63 FoV using the Ring Background model with a correlation radius of $0.5^{\circ}$ as described in Berge et al. (2007). A fit of the signal with the instrument's point spread function, i.e. a two dimensional Gaussian, gives (J2000) RA $13^{\mathrm{h}} 2^{\mathrm{m}} 41^{\mathrm{s}} \pm 6_{\text {stat }}^{\mathrm{s}}$, Dec. $-63^{\circ} 49^{\prime} 1^{\prime \prime} \pm 41_{\text {stat }}^{\prime \prime}$ for the excess center position. This is within errors compatible with the nominal position of PSR B1259-63 (Wang et al. 2004) and the TeV signal detected in 2004 (Aharonian et al. 2005b). 
Table 3. Spectral parameters for a power law fit to the monthly HESS data.

\begin{tabular}{|c|c|c|c|c|c|c|c|c|c|c|c|c|}
\hline Period & $\begin{array}{c}\overline{M J D} \\
{[\mathrm{~d}]}\end{array}$ & $\begin{array}{c}\Delta_{\overline{M J D}}^{+} \\
{[\mathrm{d}]} \\
\end{array}$ & $\begin{array}{c}\Delta_{\overline{M J D}}^{-} \\
{[\mathrm{d}]}\end{array}$ & $\tau$ & $\theta$ & $\phi$ & $\Gamma$ & $\begin{array}{c}\Phi_{0} \\
{\left[10^{-12} \mathrm{TeV}^{-1} \mathrm{~cm}^{-2} \mathrm{~s}^{-1}\right]}\end{array}$ & $\begin{array}{c}F_{E} \\
{\left[10^{-12} \mathrm{erg} \mathrm{cm}^{-2} \mathrm{~s}^{-1}\right]}\end{array}$ & $\begin{array}{c}L_{\gamma} \\
{\left[10^{32} \mathrm{erg} \mathrm{s}^{-1}\right]}\end{array}$ & $\chi^{2} / \mathrm{ndf}$ & $P_{\chi^{2}}$ \\
\hline 2004 & 53104.4 & $\ldots$ & $\ldots$ & $\ldots$ & $\ldots$ & $\ldots$ & $2.7 \pm 0.2$ & $1.3 \pm 0.1$ & $3.0 \pm 0.9$ & $8 \pm 2$ & $2.3 / 5$ & 0.81 \\
\hline 2007 & & & & & & & & & & & & \\
\hline May & 54235.1 & 5.7 & 6.3 & -74 & -0.38 & -0.060 & $2.6 \pm 0.5$ & $0.7 \pm 0.2$ & $1.4 \pm 0.4$ & $4 \pm 1$ & $2.374 / 3$ & 0.50 \\
\hline June & 54263.1 & 5.7 & 8.3 & -46 & -0.35 & -0.037 & $3.8 \pm 0.5$ & $1.5 \pm 0.2$ & $3.0 \pm 0.4$ & $8 \pm 1$ & $1.352 / 3$ & 0.72 \\
\hline July & 54291.9 & 8.0 & 7.1 & -17 & -0.25 & -0.014 & $2.7 \pm 0.3$ & $1.3 \pm 0.4$ & $2.5 \pm 0.6$ & $7 \pm 2$ & $1.294 / 3$ & 0.73 \\
\hline Total $^{* *}$ & 54262.5 & $\ldots$ & $\ldots$ & $\ldots$ & $\ldots$ & $\ldots$ & $2.8 \pm 0.2$ & $1.1 \pm 0.1$ & $2.2 \pm 0.6$ & $6 \pm 2$ & $4.678 / 5$ & 0.46 \\
\hline
\end{tabular}

For the periods April and August, no spectrum could be obtained due to low statistics: Mean Modified Julian Date $\overline{M J D}$, $\Delta_{\overline{M J D}}^{+-}$date range, days relative to periastron $\tau$, true anomaly $\theta$, orbital phase (mean anomaly) $\phi$, Photon index $\Gamma$, flux normalisation $\Phi_{0}$, mean energy flux $F_{E}$ and $\gamma$-ray luminosity $L_{\gamma}$ above $1 \mathrm{TeV}^{*}, \chi^{2}$ per number of degrees of freedom $\frac{\chi^{2}}{\mathrm{ndf}}$ and probability $P_{\chi^{2}}$ for the fit are shown. Shown are statistical errors only. ${ }^{*}$ Monthly values for $F_{E}$ and $L \gamma$ in 2007 have been computed assuming a fixed photon index of $\Gamma=2.8$.

** Entire HESS dataset from April to August 2007.

The analysis of the 2005 and 2006 datasets showed no significant excess in $\gamma$-ray events (Table 2). A calculation of upper limits at the $99 \%$ confidence level according to Feldman \& Cousins (1998) on the integrated photon flux above $1 \mathrm{TeV}$ yields $7.1 \times 10^{-13} \mathrm{~cm}^{-2} \mathrm{~s}^{-1}$ and $7.0 \times 10^{-13} \mathrm{~cm}^{-2} \mathrm{~s}^{-1}$ for these measurements, respectively.

A cross check analysis which is based on a semi-analytical model approach for air showers in order to predict the expected intensity in each pixel of the camera as described in de Naurois (2005) was also performed using an independent chain of rawdata processing. This method shows a similar signal efficiency but superior background rejection compared to the Hillas analysis. The latter has been used in the field for over 20 years now and is also more robust against systematics. Throughout this article the Hillas analysis method is used.

\subsection{Energy spectra}

A spectral analysis of the detected excess events from within the ON region for the whole 2007 dataset using the Hillas analysis shows that the differential energy spectrum of the collected photons as a function of particle energy follows a simple power law of the form

$$
\mathrm{d} N / \mathrm{d} E=\Phi_{0} \cdot(E / 1 \mathrm{TeV})^{-\Gamma}
$$

with flux normalisation at $1 \mathrm{TeV} \Phi_{0}=\left(1.1 \pm 0.1_{\text {stat }} \pm 0.2_{\text {sys }}\right) \times$ $10^{-12} \mathrm{TeV}^{-1} \mathrm{~cm}^{-2} \mathrm{~s}^{-1}$ and photon index $\Gamma=2.8 \pm 0.2_{\text {stat }} \pm 0.2_{\text {sys }}$ (see Fig. 2). The observation periods in May, June and July 2007, which allowed for an extraction of a monthly spectrum are shown in Table 3. All spectra were well described by a power law as shown by the reduced $\chi^{2}$ values. Even though there are no significant changes in photon index among the individual darkness periods from May to July, the possibility of spectral hardening towards periastron indicated when comparing e.g. $\Gamma$ and $\Phi_{0}$ between May and June in Table 3 (see also Fig. 3) was investigated. The $1 \sigma$ and $2 \sigma$ error contours of the correlated parameters $\Gamma$ and $\Phi_{0}$ taken from the power law fit to the monthly spectra are shown in Fig. 3, showing no significant evidence for spectral variability.

\subsection{Lightcurve}

The integral VHE flux of photons $F(>1 \mathrm{TeV})$ above an energy of $1 \mathrm{TeV}$ has been calculated by integrating Eq. (1) above this threshold, assuming an average photon index of $\Gamma=2.8$ taken from the overall spectrum (Fig. 2). The flux normalisation $\Phi_{0}$ has been determined as outlined in Aharonian et al. (2005b).

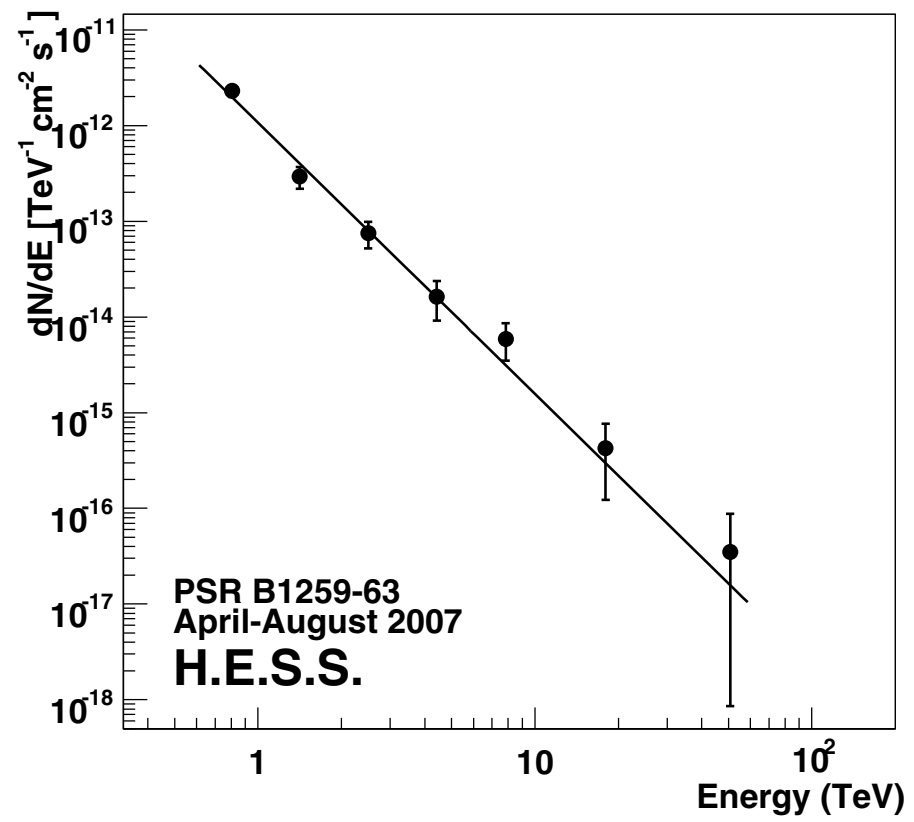

Fig. 2. Overall differential energy spectrum $\mathrm{d} N / \mathrm{d} E$ for $\gamma$-ray photons taken from within the $0.112^{\circ} \mathrm{ON}$ region around the target position of PSR B1259-63 extracted from 52.5 h (livetime) data taken between April and August 2007. The spectrum can be described by a simple power law with flux normalisation $\Phi_{0}=\left(1.1 \pm 0.1_{\text {stat }} \pm 0.2_{\text {sys }}\right) \times$ $10^{-12} \mathrm{TeV}^{-1} \mathrm{~cm}^{-2} \mathrm{~s}^{-1}$ and photon index $\Gamma=2.8 \pm 0.2_{\text {stat }} \pm 0.2_{\text {sys }}$. The integral flux for all $\gamma$-rays above $1 \mathrm{TeV}$ is $F(>1 \mathrm{TeV})=\left(6.1 \pm 0.7_{\text {stat }}\right) \times$ $10^{-13} \mathrm{~cm}^{-2} \mathrm{~s}^{-1}$.

There was no significant excess in $\mathrm{TeV} \gamma$-rays in 2005, 2006 and during the first exposures in 2007 taken in April $(\tau=-104 \mathrm{~d}$; $\tau=0 \mathrm{~d}$ being the periastron passage) which re-confirms the variable character of this source as already established from the night by night 2004 HESS data. In 2007 the total integrated photon flux above $1 \mathrm{TeV}$ is $\left(6.1 \pm 0.7_{\text {stat }}\right) \times 10^{-13} \mathrm{~cm}^{-2} \mathrm{~s}^{-1}$ corresponding to $2.7 \%$ of that of the Crab nebula above the same threshold. This translates into a mean energy flux of the VHE emission of $F_{E}(E>1 \mathrm{TeV}) \approx 2 \times 10^{-12} \mathrm{erg} \mathrm{cm}^{-2} \mathrm{~s}^{-1}$ implying a $\gamma$-ray luminosity of $L_{\gamma} \approx 6 \times 10^{32} \mathrm{erg} \mathrm{s}^{-1}$ at a distance of $1.5 \mathrm{kpc}$. We note this luminosity $\sim 0.1 \%$ of the pulsar spin-down power. The average flux levels for each observation period in 2007 are shown in the monthly lightcurve in Fig. 4. As shown in Table 2 the emission of $\mathrm{TeV}$ photons from PSR B1259-63 where a significant signal $(>5 \sigma)$ was seen under both analyses started in June 2007, i.e. $\sim 50$ days prior to the periastron passage $(\tau=-50 \mathrm{~d})$. A fit 

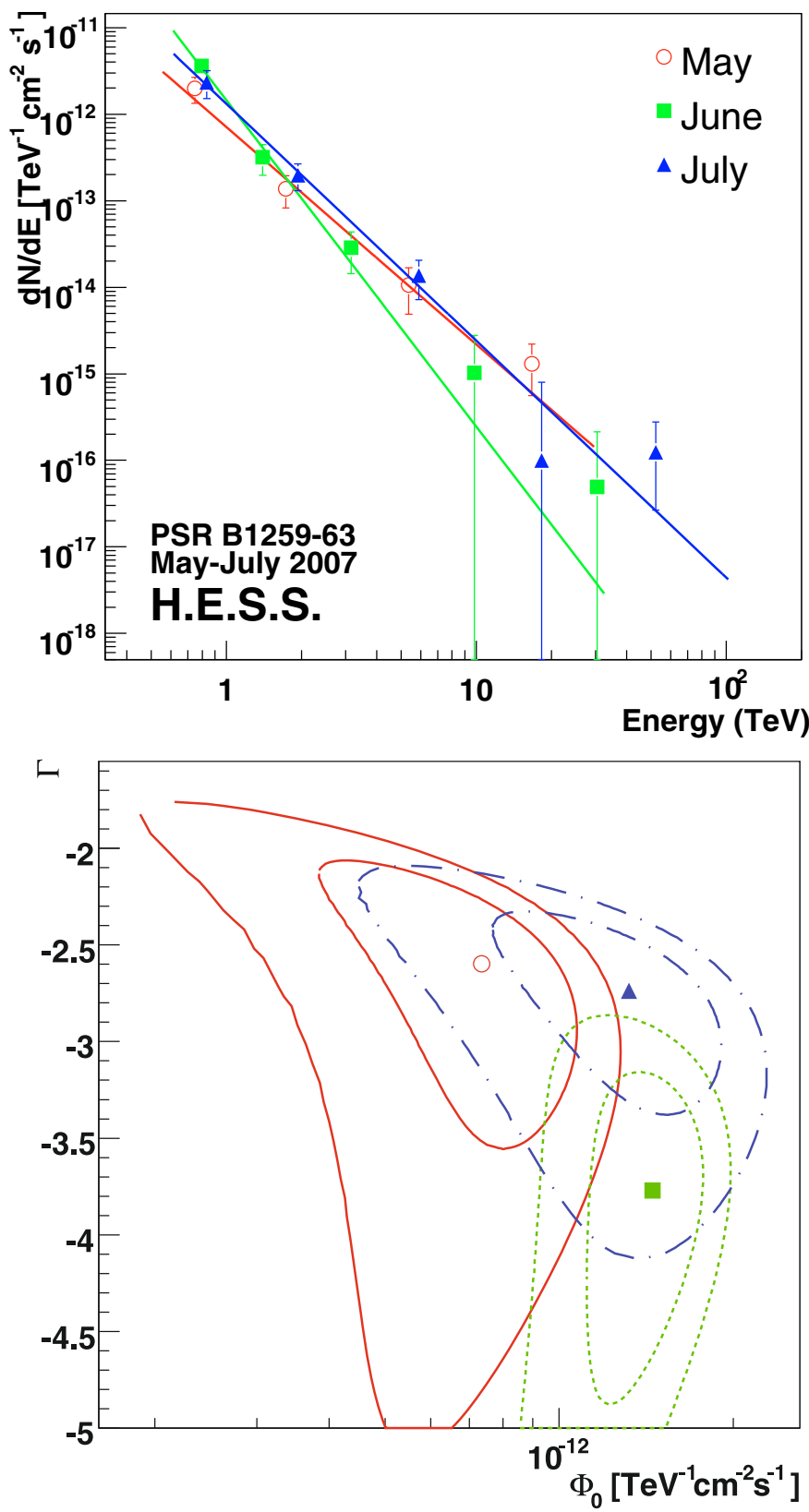

Fig. 3. (Top) PSR B1259-63 differential energy spectra $d N / d E$ for the monthly darkness periods May, June and July 2007. For the data subsets taken in April and August, no spectra could be derived due to insufficient statistics. (Bottom) $1 \sigma$ and $2 \sigma$ error contours for the correlated parameters $\Phi_{0}$ and $\Gamma$ of a power law fit to the spectra taken from the observation periods in May, June and July.

with a constant to the monthly lightcurve (April-August 2007) leads to a goodness of fit of $\chi^{2} / \mathrm{ndf}=\frac{15.7}{4}$ resulting in a probability of $P_{\chi^{2}}=3 \times 10^{-3}$.

The combination of the monthly integrated fluxes presented in Fig. 4 with the daily lightcurve as extracted from the 2004 HESS campaign (Aharonian et al. 2005b) together with the measurements from 2005 and 2006 in a single plot is shown in Fig. 5. In this representation the flux is shown as a function of the true anomaly (lower axis) and orbital phase ${ }^{2}$ (upper axis), respectively. It can be seen that the overall flux level in 2007 is comparable to that measured in 2004, while they correspond to

2 The true anomaly $\theta$ and orbital phase $\phi$ (mean anomaly) of the system vary between -0.5 and 0.5 with periastron passage defined as $\theta=\phi=0$.

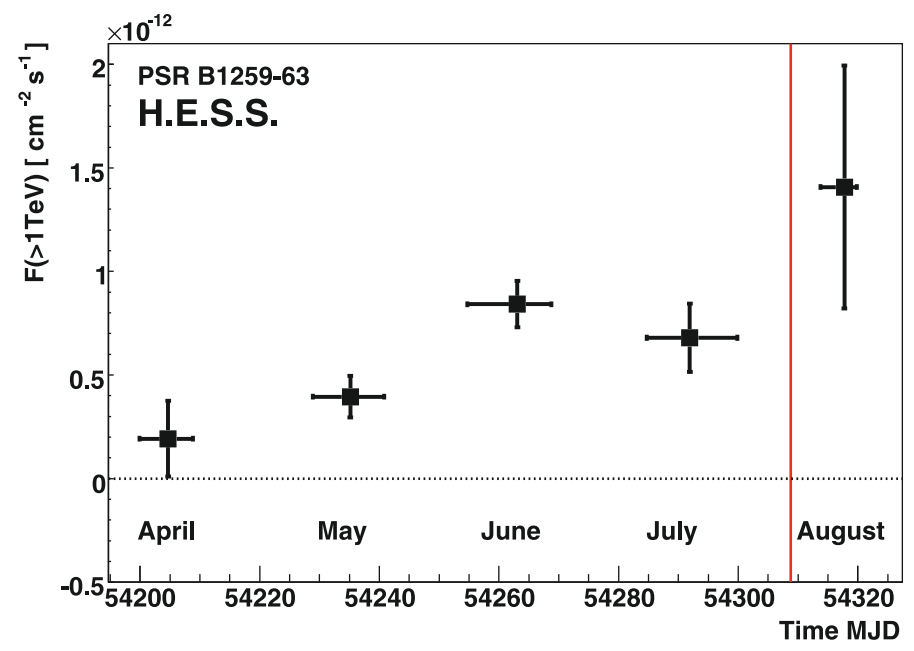

Fig. 4. Integrated photon flux above $1 \mathrm{TeV}$ for the individual HESS observation periods from April to August 2007. The red vertical line indicates the periastron passage. There was no significant $\gamma$-ray emission from PSR B 1259-63 detected in the April pointings whereas the photon flux became notable at the $3 \sigma$-level from $\sim 75$ days prior to periastron onwards. A fit with a constant to the monthly data gives $P_{\chi^{2}}=3 \times 10^{-3}$.

different orbital phases. All in all the lightcurve could be not only asymmetric with respect to periastron, also the two humps appear to exhibit a different shape with the pre-periastron hump being broader.

Between the datasets of 2004 and 2007 there is only a marginal overlap in orbital phase, i.e. the exposures taken in July and August 2007 match each one night of observations of the 2004 campaign (at a true anomaly $\theta=-0.17$ and $\theta=0.20$, respectively). The measurements are not statistically different (1.8 and 0.6 standard deviations, respectively). However, the 2007 measurements suffer from low statistics. It is not possible at present to test whether the source shows orbit-to-orbit variability and more overlapping data would be desirable.

\section{Discussion}

As with other VHE sources, the production of VHE gamma-rays in this binary requires a population of particles with multi-TeV energies. One possibility is shock acceleration within the termination zone of colliding winds (Maraschi \& Treves 1981; Tavani et al. 1994; Tavani \& Arons 1997; Bogovalov et al. 2008) which applies for PSR B1259-63/SS2883 where the PW is shocked by the stellar wind.

The modeling of the observed variability in $\mathrm{TeV}$ photon flux is based on IC scattering (Khangulyan et al. 2007) as well as hadronic interactions taking place when the pulsar interferes with the equatorial matter outflow. The latter simply explain the VHE flux variability with the phases when the pulsar crosses the dense circumstellar disc (Chernyakova et al. 2006).

\subsection{IC scenarios}

In the IC scenario many parameters contribute to the complexity of the problem. The overall system geometry significantly influences predictions for the VHE emission as shown by Kirk et al. (1999). The IC cross section varies with orbital phase as the angle between the line of sight and the vector connecting the stars changes. Also the magnetic field strength $B$ will be a function of the separation $d$ of the two objects due to changing 


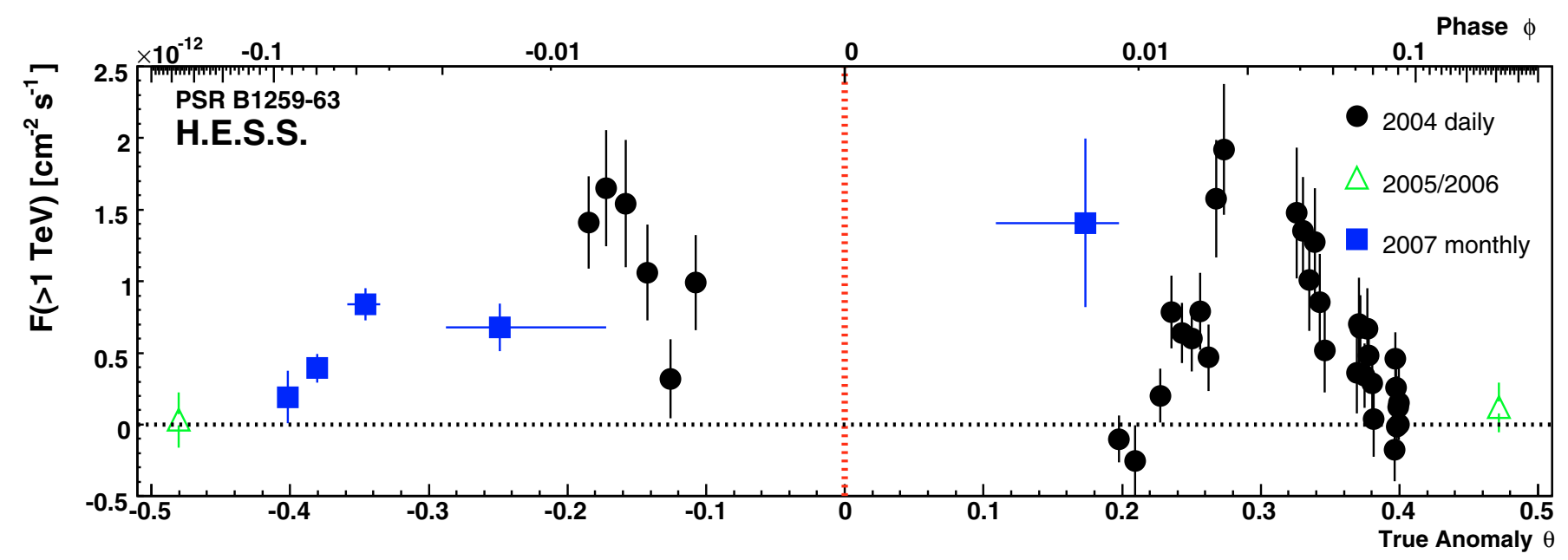

Fig. 5. VHE integrated flux from PSR B1259-63 above $1 \mathrm{TeV}$ as a function of the true anomaly. The corresponding orbital phases (mean anomaly) are shown on the upper horizontal axis. The red vertical line indicates the periastron passage. Shown are data from the years 2004 to 2007 : the black points are the daily fluxes as measured in 2004. Green empty triangles show the overall flux level as seen in 2005 and 2006 . Blue filled squares represent the monthly fluxes taken from the campaign in 2007.

magneto hydrodynamic conditions at the PW shock where the field lines from the wind get compressed (Tavani \& Arons 1997; Kirk et al. 1999). The $B$-field should become stronger towards periastron, resulting in faster synchrotron losses. This in turn means a shift in the ratio of radiation timescales, affecting the efficiency of IC cooling for VHE electrons in the Klein-Nishina regime and hence the $\mathrm{TeV}$ photon index. Moreover non-radiative cooling mechanisms such as adiabatic expansion of the shock region or particle escape can be important effects as demonstrated by Khangulyan et al. (2007). In Bogovalov et al. (2008) it was pointed out that the interaction of pulsar and stellar winds leads to relativistic motion of matter at the termination shock. The corresponding Doppler factor with respect to the line of sight will strongly depend on the position of the pulsar along its orbit causing modulations of the non-thermal radiation of electrons.

Finally, Bednarek (1997), Sierpowska-Bartosik \& Bednarek (2008) and Bosch-Ramon et al. (2008) have discussed the possibility of radiating secondaries stemming from pair cascades forming close to the star where the photon field density is high enough.

\subsection{Hadronic disc scenarios}

Concerning hadronic scenarios, the stellar disc seems to be an ideal reservoir for target material interacting with the PW. In this regard parameters like the disc's density, thickness, extension and orientation with respect to the pulsar orbit are crucial. Currently, however, the knowledge about these quantities is limited and depends on model interpretations of the available radio to X-ray data as discussed in Johnston et al. (1994, 1996, 2005), Melatos et al. (1995), Tavani \& Arons (1997) and Bogomazov (2005). According to these studies the disc appears to be inclined by an angle $\delta$ in the range of $10^{\circ}-40^{\circ}$ compared to the pulsar ecliptic. Moreover the vanishing of the pulsed radio signal between 16 days before and 15 days after periastron (Johnston et al. 2005) accounts for the asymmetric position of the disc's line of intersection with the orbital plane with respect to the orbital semimajor axis. In Chernyakova et al. (2006), the disc position is inferred from the HESS 2004 data by assuming that the peak VHE emission corresponds to orbital phases of maximum circumstellar density. According to this, the disc intersects the pulsar orbit at a true anomaly of $\theta=-0.197$ and $\theta=0.303$, respectively $(\theta \in[-0.5,0.5])$ with respect to periastron and covers the pulsar orbit over an angle band of $\sim 18.5^{\circ}(\Delta \theta=0.05)$.

\subsection{Interpretation of the 2007 VHE data}

Looking at the above estimates for the disc location of SS2883, the integrated flux data presented here are difficult to understand within a purely hadronic disc scenario, considering a simple symmetry argument. According to this the excess in June (Table 2), corresponding to 47 days prior to periastron passage, occurs at unexpectedly small values for the true anomaly of $\theta \approx-0.35\left(\approx-50^{\circ}\right.$ off the expected disc density maximum; see Fig. 6), inconsistent with the position of the Be disc as computed by Chernyakova et al. (2006) from the $2004 \mathrm{TeV}$ data alone (see their Fig. 4). Following their approach and fitting the complete data set including the measurements in 2007 with a Gaussian gives a very poor $\chi^{2} /$ ndf of $118.2 / 39$, in contrast to $40.0 / 34$ for a fit omitting the 2007 data as shown in Fig. 7 . In this approach, flux data gathered after periastron passage has been shifted with respect to periastron by 0.5 and added to the preperiastron phase. This is based on the simple assumption that the position of the second crossing should be shifted by $180^{\circ}$ relative to the first entrance of the pulsar into the disc. Note, however, that in this approach it has not been considered that the binary separation is different for the corresponding pulsar positions when being shifted, translating into different densities in the stellar disc.

The above argument is also supported by the discrepancy between the disc parameters resulting from this approach and the eclipse of the pulsed radio emission. The pulsed signal emerges 10 days earlier from the eclipse than would be expected (Dubus 2006b) and the eclipse itself is slightly shifted towards preperiastron phases which in addition contradicts the above disc location. Altogether this would either suggest a complicated disc morphology, e.g., propagating bubbles of disc material, or reflecting the fact that the disc cannot be the only explanation for the onset of VHE radiation, thus favoring disc independent scenarios such as the IC upscattering of stellar photons at least as additional origins for the $\mathrm{TeV}$ emission. 


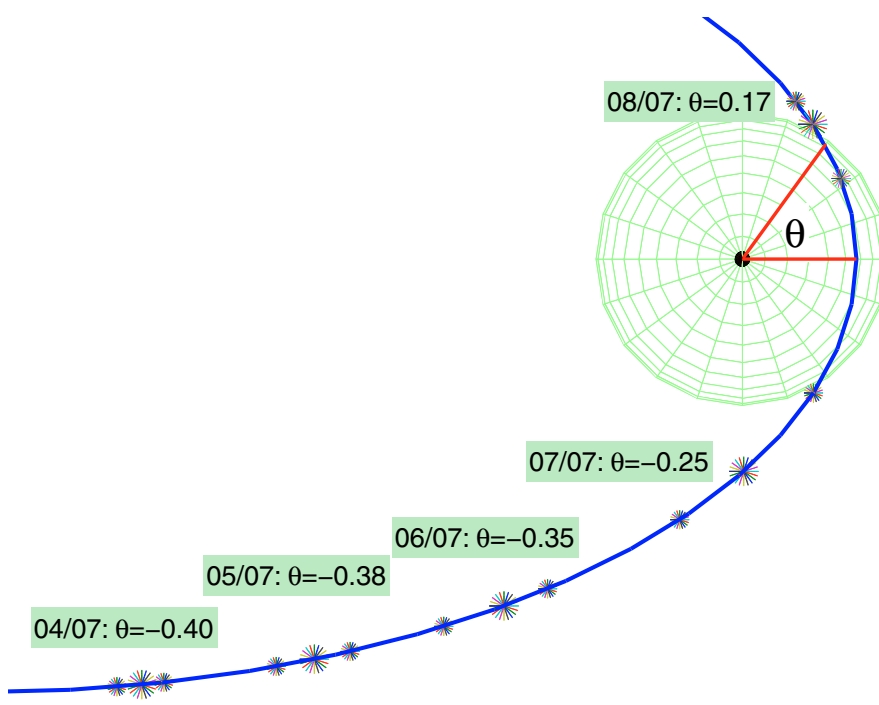

Fig. 6. Top view model sketch for the PSR B1259-63/SS2883 orbit. The black dot represents SS2882 and the circumstellar disc is depicted in green. The pulsar size is exaggerated by a huge factor. The circumstellar disc is assumed to be extended out to 20 stellar radii $R_{\odot}$, as typical for Be stars. The mean pulsar positions for the individual HESS observation periods in 2007 are shown along the orbital trajectory as stars together with the corresponding date and the true anomaly $\theta$ (definition indicated by red thin lines). The smaller stars indicate the pulsar position of each period's first and last measurement, respectively. The positive excess of $\mathrm{TeV}$ photons in June 2007 at an orbital position of $\theta \approx-0.35$ seems to have occurred far outside the estimated disc crossing phase.

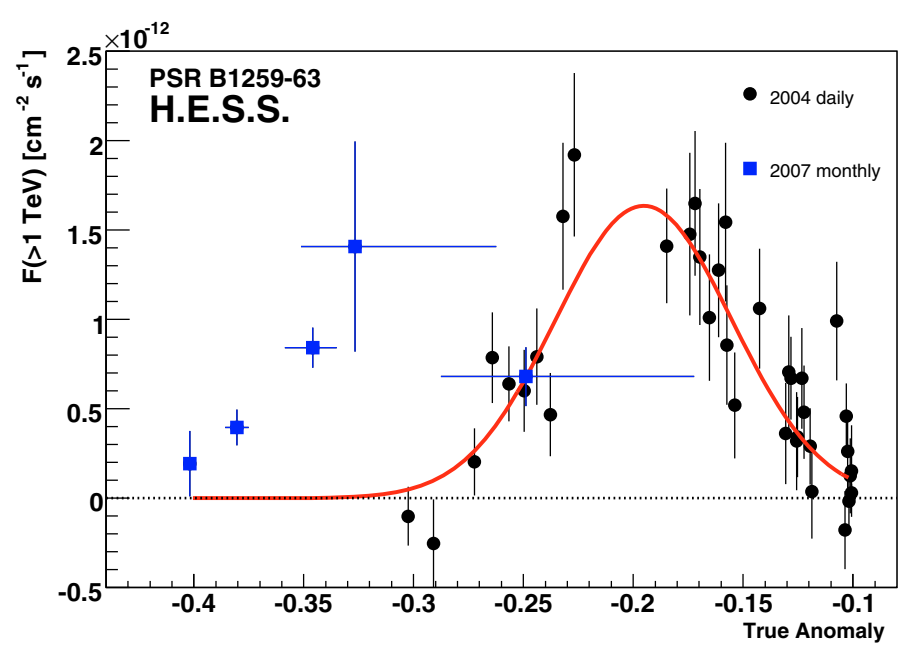

Fig. 7. Fit of the 2004 (black points) integrated flux data above $1 \mathrm{TeV}$ with a Gaussian following Fig. 4 in Chernyakova et al. (2006) in order to determine the location of the circumstellar disc in comparison with fluxes measured in 2007 (blue squares). The 2007 data deviates considerably from the suggested disc density model.

\section{Conclusions}

The second HESS campaign on the binary system PSR B 1259-63/SS2883 around periastron in 2007 $(-0.08<\phi<0.01)$ yielded VHE data for the first time covering early pre-periastron orbital phases commencing $\sim 110$ days before the passage. Measurements in $2005(\phi=0.31)$ and $2006(\phi=-0.37)$ covering orbital phases near apastron for the first time showed no significant flux of $\gamma$-rays from this source leading to an upper limit of $7 \times 10^{-13} \mathrm{~cm}^{-2} \mathrm{~s}^{-1}$ for the integrated photon flux above $1 \mathrm{TeV}$ at the respective orbital positions. As expected the source showed a significant excess $(9.5 \sigma)$ of $\mathrm{TeV}$ $\gamma$-rays in 2007 after 3.4 years of quiescence. Spectral features as well as the variable source character known from exposures in 2004 were confirmed. The mean energy flux of the source was measured to be $F_{E}(E>1 \mathrm{TeV}) \approx 2 \times 10^{-12} \mathrm{erg} \mathrm{cm}^{-2} \mathrm{~s}^{-1}$ corresponding to an integrated flux in VHE photons above $1 \mathrm{TeV}$ of about $2.7 \%$ of that of the Crab nebula. The overall lightcurve including also data taken in 2004 shows two 'humps' located at phases $\phi \approx-0.08 \ldots-0.004$ and $\phi \approx 0.009 \ldots 0.08$ with peak emissions of significant photon excesses at $\phi \approx-0.007$ and $\phi \approx 0.017$, respectively. This appears somewhat different to the VHE flux vs. $\phi$ trend seen in the moderate-length period VHE binary LSI+61 303, which exhibits VHE emission over a wide range of orbital phases $\phi \approx 0.225 . .0 .625$ and a peak at $\phi \approx 0.325 . .0 .425$ (Albert et al. 2009) $)^{3}$. However, LSI+61 303 is a much more compact system than PSR B1259-63: the compact object in LSI+61 303 is always at a distance from its Be companion that is smaller than or equal to the orbital separation at periastron passage in PSR B1259-63. The early onset of the VHE emission in PSR B1259-63 with respect to the pulsar's orbital phase ( $\phi \approx-0.04)$ occurs much further away from the Be star. This presents difficulties for a pure hadronic disc scenario taking into account positional parameters for the circumstellar disc. Thus, models which associate the TeV emission in PSR B1259-63 with the pulsar crossing the disc are clearly challenged by the HESS data presented in this article.

PSR B1259-63 remains a fascinating TeV binary system featuring complex PW dynamics with many questions still open. More sensitive observations in the VHE regime using future observatories that would allow for phase resolved spectra with time apertures on time scales of days, could contribute to a better understanding of this source.

Acknowledgements. The support of the Namibian authorities and of the University of Namibia in facilitating the construction and operation of HESS is gratefully acknowledged, as is the support by the German Ministry for Education and Research (BMBF), the Max Planck Society, the French Ministry for Research, the CNRS-IN2P3 and the Astroparticle Interdisciplinary Programme of the CNRS, the UK Science and Technology Facilities Council (STFC), the IPNP of the Charles University, the Polish Ministry of Science and Higher Education, the South African Department of Science and Technology and National Research Foundation, and by the University of Namibia. We appreciate the excellent work of the technical support staff in Berlin, Durham, Hamburg, Heidelberg, Palaiseau, Paris, Saclay, and in Namibia in the construction and operation of the equipment. We would like to thank Masha Chernyakova for fruitful discussions.

\section{References}

Aharonian, F., Akhperjanian, A. G., Aye, K.-M., et al. 2005a, Science, 309, 746 Aharonian, F., Akhperjanian, A. G., Aye, K.-M., et al. 2005b, A\&A, 442, 1 Aharonian, F., Akhperjanian, A. G., Aye, K.-M., et al. 2005c, A\&A, 439, 1013 Aharonian, F., Akhperjanian, A. G., Bazer-Bachi, A. R., et al. 2006, A\&A, 457, 899

Albert, J., Aliu, E., Anderhub, H., et al. 2006, Science, 312, 1771

Albert, J., Aliu, E., Anderhub, H., et al. 2007, ApJ, 665, L51

Albert, J., Aliu, E., Anderhub, H., et al. 2009, ApJ, 693, 303

Aragona, C., McSwain, M. V., Grundstrom, E. D., et al. 2009, ApJ, 698, 514

Ball, L., \& Kirk, J. G. 2000, Astrop. Phys., 12, 335

Bednarek, W. 1997, A\&A, 322, 523

Berge, D., Funk, S., \& Hinton, J. 2007, A\&A, 466, 1219

Bogomazov, A. I. 2005, Astron. Rep., 49, 709

Bogovalov, S. V., Khangulyan, D. V., Koldoba, A. V., Ustyugova, G. V., \& Aharonian, F. A. 2008, MNRAS, 387, 63

Bosch-Ramon, V., Khangulyan, D., \& Aharonian, F. A. 2008, A\&A, 482, 397 Chernyakova, M., Neronov, A., Lutovinov, A., Rodriguez, J., \& Johnston, S. 2006, MNRAS, 367, 1201

3 Here we quote phases subtracting the presently defined periastron phase at $\phi^{*}=0.275$ (Aragona et al. 2009). 
de Naurois, M. 2005, in Towards a Network of Atmospheric Cherenkov Detectors VII, Palaiseau, ed. B. Degrange, \& G. Fontaine, 149

Dubus, G. 2006a, A\&A, 456, 801

Dubus, G. 2006b, Frontier Objects in Astrophysics and Particle Physics, ed. F. Giovannelli, \& G. Mannocchi, Italian Physical Society, Editrice Compositori, Bologna, Italy, 93, 273

Feldman, G. J., \& Cousins, R. D. 1998, Phys. Rev. D, 57, 3873

Funk, S., Hermann, G., Hinton, J., et al. 2004, Astropart. Phys., 22, 285

Greiner, J., Tavani, M., \& Belloni, T. 1995, ApJ, 441, L43

Hirayama, M., Cominsky, L. R., Kaspi, V. M., et al. 1999, ApJ, 521, 718

Johnston, S., Manchester, R. N., Lyne, A. G., et al. 1992, ApJ, 387, L37

Johnston, S., Manchester, R. N., Lyne, A. G., Nicastro, L., \& Spyromilio, J. 1994, MNRAS, 268, 430

Johnston, S., Manchester, R. N., Lyne, A. G., et al. 1996, MNRAS, 279, 1026

Johnston, S., Manchester, R. N., McConnell, D., \& Campbell-Wilson, D. 1999, MNRAS, 302, 277

Johnston, S., Ball, L., Wang, N., \& Manchester, R. N. 2005, MNRAS, 358, 1069

Kawachi, A., Naito, T., Patterson, J. R., et al. 2004, ApJ, 607, 949

Khangulyan, D., Hnatic, S., Aharonian, F., \& Bogovalov, S. 2007, MNRAS, 380, 320

Kirk, J. G., Ball, L., \& Skjaeraasen, O. 1999, Astropart. Phys., 10, 31

Li, T.-P., \& Ma, Y.-Q. 1983, ApJ, 272, 317

Maraschi, L., \& Treves, A. 1981, MNRAS, 194, 1P

Melatos, A., Johnston, S., \& Melrose, D. B. 1995, MNRAS, 275, 381

Neronov, A., \& Chernyakova, M. 2007, Ap\&SS, 309, 253

Nicastro, L., Dal Fiume, D., Orlandini, M., et al. 1999, Nucl. Phys. B Proc. Suppl., 69, 170

Shaw, S. E., Chernyakova, M., Rodriguez, J., et al. 2004, A\&A, 426, L33

Sierpowska-Bartosik, A., \& Bednarek, W. 2008, MNRAS, 385, 2279

Tavani, M., \& Arons, J. 1997, ApJ, 477, 439

Tavani, M., Arons, J., \& Kaspi, V. M. 1994, ApJ, 433, L37

Uchiyama, Y., Tanaka, T., Takahashi, T., Mori, K., \& Nakazawa, K. 2009, ApJ

Wang, N., Johnston, S., \& Manchester, R. N. 2004, MNRAS, 351, 599

1 Max-Planck-Institut für Kernphysik, PO Box 103980, 69029

Heidelberg, Germany

2 Yerevan Physics Institute, 2 Alikhanian Brothers St., 375036

Yerevan, Armenia

3 Centre d'Étude Spatiale des Rayonnements, CNRS/UPS, 9 Av. du

Colonel Roche, BP 4346, 31029 Toulouse Cedex 4, France

4 Universität Hamburg, Institut für Experimentalphysik, Luruper

Chaussee 149, 22761 Hamburg, Germany

5 Institut für Physik, Humboldt-Universität zu Berlin, Newtonstr.

15, 12489 Berlin, Germany

e-mail: mkersch@physik.hu-berlin.de

6 LUTH, Observatoire de Paris, CNRS, Université Paris Diderot, 5

Place Jules Janssen, 92190 Meudon, France

7 IRFU/DSM/CEA, CE Saclay, 91191 Gif-sur-Yvette, Cedex, France

${ }^{8}$ University of Durham, Department of Physics, South Road, Durham DH1 3LE, UK
9 Unit for Space Physics, North-West University, Potchefstroom 2520, South Africa

${ }^{10}$ Laboratoire Leprince-Ringuet, École Polytechnique, CNRS/IN2P3, 91128 Palaiseau, France

11 Laboratoire d'Annecy-le-Vieux de Physique des Particules, Université de Savoie, CNRS/IN2P3, 74941 Annecy-le-Vieux, France

12 Astroparticule et Cosmologie (APC), CNRS, Université Paris 7 Denis Diderot, 10 rue Alice Domon et Leonie Duquet, 75205 Paris Cedex 13, France UMR 7164 (CNRS, Université Paris VII, CEA, Observatoire de Paris), France

${ }_{13}$ Dublin Institute for Advanced Studies, 5 Merrion Square, Dublin 2, Ireland

${ }^{14}$ Landessternwarte, Universität Heidelberg, Königstuhl, 69117 Heidelberg, Germany

${ }^{15}$ Laboratoire de Physique Théorique et Astroparticules, Université Montpellier 2, CNRS/IN2P3, CC 70, Place Eugène Bataillon, 34095 Montpellier Cedex 5, France

16 Universität Erlangen-Nürnberg, Physikalisches Institut, ErwinRommel-Str. 1, 91058 Erlangen, Germany

17 Laboratoire d'Astrophysique de Grenoble, INSU/CNRS, Université Joseph Fourier, BP 53, 38041 Grenoble Cedex 9, France

18 Institut für Astronomie und Astrophysik, Universität Tübingen, Sand 1, 72076 Tübingen, Germany

19 LPNHE, Université Pierre et Marie Curie Paris 6, Université Denis Diderot Paris 7, CNRS/IN2P3, 4 Place Jussieu, 75252 Paris Cedex 5, France

20 Charles University, Faculty of Mathematics and Physics, Institute of Particle and Nuclear Physics, V Holešovičkách 2, 180 00, Czech Republic

21 Institut für Theoretische Physik, Lehrstuhl IV: Weltraum und Astrophysik, Ruhr-Universität Bochum, 44780 Bochum, Germany

22 University of Namibia, Private Bag 13301, Windhoek, Namibia

23 Obserwatorium Astronomiczne, Uniwersytet Jagielloński, ul. Orla 171, 30-244 Kraków, Poland

${ }^{24}$ Nicolaus Copernicus Astronomical Center, ul. Bartycka 18, 00716 Warsaw, Poland

${ }^{25}$ School of Physics \& Astronomy, University of Leeds, Leeds LS2 9JT, UK

26 School of Chemistry \& Physics, University of Adelaide, Adelaide 5005, Australia

27 Toruń Centre for Astronomy, Nicolaus Copernicus University, ul. Gagarina 11, 87-100 Torun, Poland

28 Instytut Fizyki Jạdrowej PAN, ul. Radzikowskiego 152, 31-342 Kraków, Poland

29 European Associated Laboratory for Gamma-Ray Astronomy, jointly supported by CNRS and MPG, Europe 